\title{
Routing Communication Inside Ad Hoc Drones Network
}

\author{
https://doi.org/10.3991/ijim.v15i17.19179 \\ Hamza Zemrane ${ }^{1(\bowtie)}$, Youssef Baddi², Abderrahim Hasbi ${ }^{1}$ \\ 'Mohammed V University, Rabat, Morocco \\ ${ }^{2}$ Chouaib Doukkali University, El Jadida, Morocco \\ zemranehamza93@gmail.com
}

\begin{abstract}
The world knows a constant development of technology applied in different sectors of activities: health, factories, homes, transportation, and others, one of the big axes that take a lot of attention today is the drone's field. To communicate information a fleet of drones can use different communication architectures: centralized communication architecture, satellite communication architecture, cellular network communication architecture and a specific Ad hoc communication architecture called the UAANET drones architecture. In our work we focused specifically on the routing of information inside the UAANET where we analyze and compare the performances of the reactive protocol AODV and the proactive protocol OLSR, when the UAANET use an applications based on the HTTP protocol, the database queries, voice application, and video conferencing application.
\end{abstract}

Keywords - drones communication architectures, UAANET drones network, routing protocols, optimized link state routing protocol (OLSR), Ad hoc on demand distance vector (AODV), Ad hoc network

\section{Introduction}

Thanks to advances in the miniaturization of on-board systems, drones called Unmanned Aerial Vehicles (UAVs) have appeared and allow civilian applications to be carried out at lower costs. To improve their performance on complex missions (for example, to bypass an obstacle), it is possible to deploy a fleet of cooperative drones in order to share tasks between the drones. This type of operation requires a high level of cooperation between the drones and the control station. Communication between the drones of the fleet is therefore an important issue in the performance of the operations of a fleet of drones. Among the different communication architectures that exist, the Ad hoc network is proving to be an efficient and promising solution for the operation of a fleet of drones. An Ad hoc network of drones called UAANET network is an autonomous system made up of a fleet of drones and one or more ground stations. This network can be considered as a sub-category of an Ad hoc mobile network with specific characteristics (high speed of the nodes, specific mobility model, etc.) which can lead to performance drops in the routing protocol used. Our role is to compare the performance 
of two different types of routing protocols, in our UAANET network, the reactive protocol AODV, and the proactive protocol OLSR protocol, when the drones are routing information of type: HTTP, voice application, video conferencing application, and database queries. In the section two we talk about the possible communication architectures for a fleet of drones, the centralized communication architecture, the satellite communication architecture, the cellular network communication architecture, the Ad hoc communication architecture, and the UAANET network of drones. In the section three we describe the routing in the UAANET network of drones, and define the hierarchical protocol, the reactive protocol where we describe the operation mode of the AODV protocol, the proactive protocol, and we describe the operation mode of the OLSR protocol, and the geographic protocol. In the section four we make a simulation of an UAANET that contain ten drones on movement, and we compare the routing protocol AODV and OLSR, and analyze their performances.

\section{Types of networks architectures used in a fleet of drones}

\subsection{Network architecture based on a central communication}

This architecture [1] is based on a wireless network that connects the set of drones and the base station on the ground. In this situation, each drone has a direct link with the station located on the floor to send the data collected by the drone and receive the commands from the central station. Direct communication between drones is not possible, to communicate with each other this must be done through the base station. In this case, the ground station acts as a relay node. An illustration of this architecture is shown in the figure below.

\subsection{Network architecture based on satellite communication}

Another type of centralized network architecture that can be envisaged for establishing communication between different drones is the deployment of a communication satellite [2]. In this configuration, the satellite functions as a communication relay. Its receiving antennas receive signals emitted from the ground station, these signals are then transposed into frequency and amplified before being retransmitted to the drones. There are two types of satellites that can be used: the geostationary satellite and the orbital satellite. An illustration of this architecture is shown in the Figure 1.

- The geostationary satellite [3] is located in the equatorial plane. It spins at the same speed and in the same direction as the earth, so its trajectory is fixed above a point on the ground.

- Orbital satellite [4] is a satellite that covers different geographic areas.

The advantage of using satellites is that they provide more effective coverage than centralized communication. This allows for improved interconnection of the drones communication network, regardless of their trajectories. 


\subsection{Cellular network communication architecture (semi-centralized network)}

This type of network [5] is based on a network of cells that are identified by base stations, this base stations are placed based on users density and the area to cover. At the level of each cell there are a number of drones and their own base station. In this type of network, communication between drones can be done by default, but only within a cell. It is also possible to extend the scope of the mission through the deployment of several stations. These stations can offer several communication links which allow, in the event of degradation of performance on a given link, to use another one. An illustration of this architecture is shown in the Figure 1.

\subsection{Ad hoc communication architecture}

The wireless communication called Ad hoc is based on a set of mobile units which have one or more wireless modules, to create a network depending on the applications used [6], these units forming the network can enter and leave at any time. Our network can therefore form and organize itself dynamically without the need for a central station or even a network infrastructure. If a drone wishes to communicate with another which is not on the same scope, the communication is done through the other drones which connect between them, the routing of the information adapts automatically according to the change of the topology. Unlike the traditional wireless network, the network service area is the geographic area in which the nodes are distributed. The wireless Ad hoc network allows communication between two nodes that are out of direct range of each other. An illustration of this architecture is shown in the Figure 1. And in the Table 1 we compare different types of application flows for every architecture.

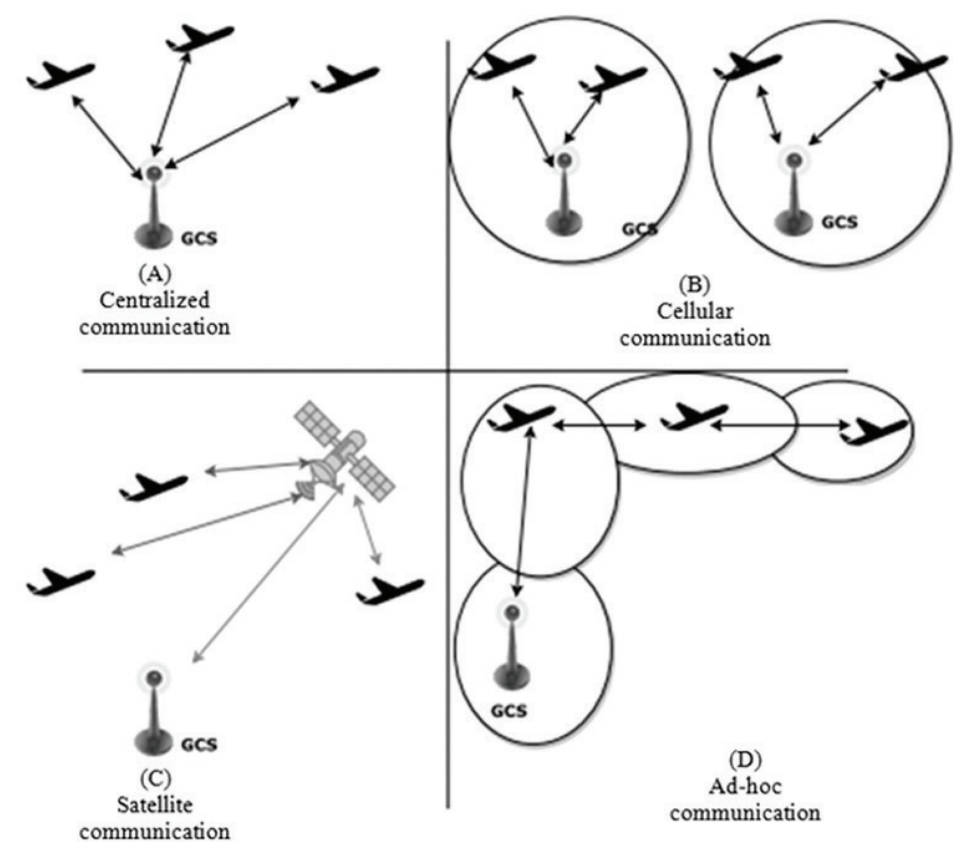

Fig. 1. Main communication architectures enabling communication for drone fleets 


\subsection{Analysis of the different communication architectures}

So for a drone fleet we have a certain number of network architecture, in the following part we distinguish the advantages of the Ad hoc architecture

- Communication with good quality between drones: the messages exchanged between drones are transmitted in real time, the communication topology can change at any time unpredictably, drones can leave the Ad hoc network and others can enter, the information routed must not be interrupted when it's routed to another drones, the network must automatically adapt to the change in the topology.

- No fixed infrastructure: the development of communication networks goes from wired networks, to wireless networks with infrastructure, to wireless networks without infrastructure [6], all the drones forming the Ad hoc network are responsible for the routing of the information and stability in the network.

- Ad hoc networks are distinguished by flexibility and mobility: depending on the mission, research drones move freely in space, communication can be bidirectional, or unidirectional, drones have the right to exit or enter the network freely.

- As opposed to centralized and cellular networks, Ad hoc networks have localized security on drones, as a result the network does not have a central point of vulnerability, the messages exchanged are controlled by the authentication and secure routing mechanisms.

\subsection{Mobility model for Ad hoc network of drones}

With regard to the UAANET network or even Flying Ad hoc NETwork (FANET) [7], the mobility model generally depends on various parameters. It is most often predictable, but in the majority of cases it is dynamically modified due to the speed of drones, climatic conditions and many other geographic and topographic parameters. A topology of an Ad Hoc network of drones is shown in the Figure 2. Indeed, we may encounter some cases of drone fleet applications with predefined or preferred trajectories. However, as the environment is dynamic, the flight plan often has to be recalculated by the autopilot. A few mobility models have been proposed in the literature. In, a PPRZM mobility model (PaPaRaZzi Mobility) it was suggested.

This model was defined with the objective of reproducing movements in a network simulator to create a more realistic movement of the nodes, which consists of the following main movements:

- Rectilinear movement: rectilinear movement of a point towards a destination position. The knot can go straight back and forth.

- Circular movement: the drone flies over a given area, following a circular path. It is defined by the position of the center and the radius that surrounds the target area.

- Oblong movement: it consists of rectilinear round trips, offset between two fixed points, with a half-turn once each point has been crossed.

- Eight movement: it is quite similar to the oblong movement. The only difference is in the crossing of their round trips. 


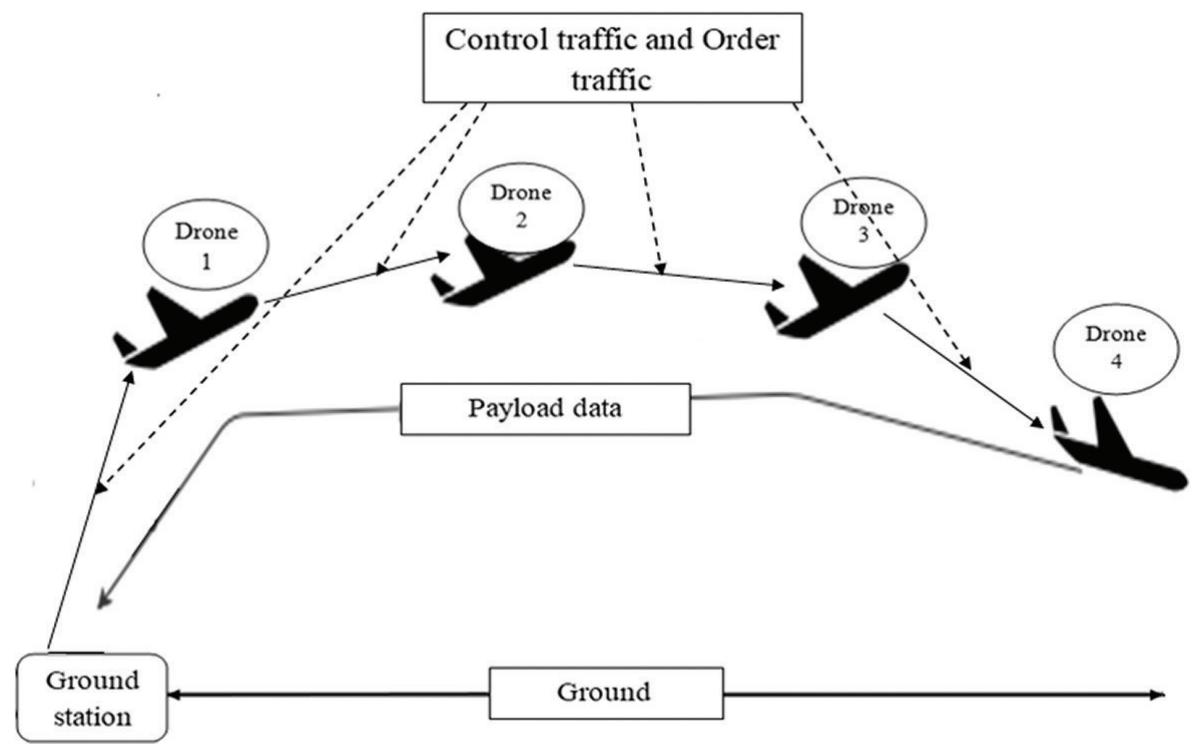

Fig. 2. Ad hoc network of drones

Table 1. Different types of application flows in an Ad hoc network of drones

\begin{tabular}{|c|c|c|c|c|}
\hline & Centralized & Satellite & Cellular & Ad hoc \\
\hline Benefits & $\begin{array}{ll}- & \text { Service } \\
& \text { discovery } \\
- & \text { Ability to control } \\
& \text { entry and exit of } \\
& \text { knots }\end{array}$ & - Connectivity & $\begin{aligned} &- \text { Connectivity } \\
&- \text { Scaling } \\
& \text { according to the } \\
& \text { number of base } \\
& \text { stations } \\
&- \text { Possibility to } \\
& \text { choose the best } \\
& \text { link among those } \\
& \text { located between } \\
& \text { the base stations }\end{aligned}$ & $\begin{array}{l}-\quad \text { Easy to deploy } \\
-\quad \text { Takes into account } \\
\text { the mobility of the } \\
\text { nodes } \\
- \text { Autonomous } \\
\text { communication } \\
\text { without } \\
\text { infrastructure } \\
-\quad \text { Communication } \\
\text { possible between } \\
\text { neighbors }\end{array}$ \\
\hline Disadvantages & $\begin{aligned} &- \text { Exchange } \\
& \text { latency between } \\
& \text { two nodes } \\
&- \text { Signal blocking } \\
& \text { possible } \\
&- \text { Bandwidth } \\
& \text { consumption } \\
&-\quad \text { Safety depends } \\
& \text { on one point }\end{aligned}$ & $\begin{aligned}- & \text { Exchange } \\
& \text { latency between } \\
& \text { two nodes } \\
- & \text { High emission } \\
& \text { power } \\
- & \text { High cost } \\
- & \text { Safety depends } \\
& \text { on one point }\end{aligned}$ & 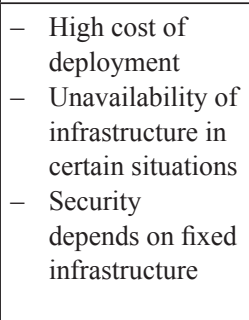 & 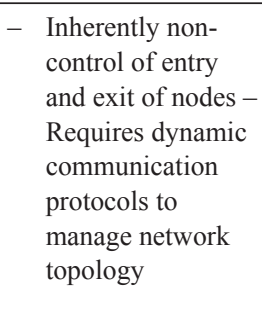 \\
\hline
\end{tabular}




\section{Communication in an Ad hoc network of drones}

\subsection{Data link (medium access control)}

The role of the link layer is to make point-to-point transmission more reliable in order to meet the latency requirements of data packets having different priorities. It is also responsible for the creation of frames, error control and admission control of the various services for the scheduling of priority packets. In addition, apart from the problem of quality of service, the MAC layer is also responsible for adopting a strategy of saving energy and network resources in the management of transmission powers and thus allows the use of the available bandwidth. A dynamic transmission range and power allocation mechanism can be implemented.

Among the Ad hoc link-level protocols we have the IEEE 802.11 family. This choice is justified for a simplified use in a context of Ad hoc multisaut communication.

\subsection{Routing in UAANET network of drones}

Routing is the mechanism by which paths are selected in a network to route data from a sender to one or more recipients. Routing is the mechanism by which paths are selected in a network to route data from a sender to one or more recipients. In UAANET networks [7] we can distinguish four main families of routing protocols, hierarchical routing protocols, reactive routing protocols where we take the AODV protocol, proactive routing protocols, where we take the OLSR protocol, and the family of geographic routing protocols.

Hierarchical protocol. Hierarchical routing is considered to be the most favorable approach in terms of energy efficiency [8]. Hierarchical protocols restructure the global network into groups called Cluster Heads, each of which consists of a leader called Cluster Head and its members. Depending on the application, members may or may not be direct neighbors of the leader and route their messages to their leader, who then routes them across the entire network via other $\mathrm{CHs}$ to the Base Station. The strong point of this type of protocol is the aggregation and merging of data in order to reduce the number of messages transmitted to the sink, which implies better energy savings. The problem that can arise in this topology is the overload of $\mathrm{CHs}$ which induces an imbalance of energy consumption in the network. To remedy this problem, CHs can be specific sensors with more energy resources and more processing capacities or they can be dynamically elected and thus guarantee a balance of energy consumption and increase fault tolerance.

Reactive protocol. Also called "On-demand" [9], establish (from a node given) a route to a destination ( $\mathrm{s}$ ) only when a node initiates a process called: "route discovery process". Once this route has been established, it will therefore be kept in the routing table until the destination is no longer accessible or the time for establishing the routes (configured by the protocol) is considered to have expired. We take as examples reactive routing protocols: AODV. 
Ad hoc on demand distance vector $(A O D V)$. AODV is a reactive routing protocol using a route discovery and route maintenance mechanism described above [10].

- Once the route is mapped out, nodes that have not been contacted will not participate in the exchange or updating of the route.

- For route maintenance, link breaks are detected using specific Hello messages broadcast periodically from a node to its immediate neighbors or by listening for the transmission of a data packet on the next link.

- The node detecting a link break broadcasts an error message using the Route Error (RERR) packet containing the address of the unreachable destination.

Proactive protocol. Protocols in this category are based on classical link state and distance vector algorithms [11]. The basic principle of these protocols is to keep routing tables up to date, by setting up a system for the periodic exchange of control packets. This way of proceeding allows the nodes to construct the topology of the network in a distributed fashion. There are two types of control packets for this purpose: packets sent locally (one-hop) for neighborhood discovery and packets broadcast throughout the network to communicate neighborhood status information to other nodes ( generally the set of neighbors or a subset) gathered by the first type of control messages.

Optimized link state routing protocol (OLSR). The OLSR protocol is based on a "link state" type algorithm [12]. To avoid the flooding of routing packets which can cause congestion, the protocol is based on the selection of specific MPR (MultiPoint Relay) standard nodes responsible for transmitting topology information [13]. The selection of these MPR nodes is made from Hello messages to deduce the nature of the links, symmetrical or asymmetrical, which connect them. The condition to be able to be MPR is to reach, with a symmetrical link, all the neighboring nodes located at a distance of two hops from the initial node. The set of MPR nodes must therefore cover the entire neighborhood located two hops away.

Geographic protocol. A routing is said to be geographic when the routing decisions are based on the position of the nodes [14]. This type of routing uses information about the location or location of sensor nodes. Generally, this information is necessary to calculate the distance between two given nodes, and therefore to estimate the power required to send the packets. The distance between two neighboring nodes can be estimated from the power of the signal on reception. The coordinates of the neighboring nodes are also obtained by exchanging information between them. The position of the nodes can be retrieved directly using positioning systems, such as the GPS (Global Position System) [15]. This routing technique consists in routing the information to the node for which it is sought after having obtained the zone in which it is located.

\section{Simulation and performance analysis}

The following simulation represents an UAANET network [7], that contains ten drones in motion, and exchanging HTTP, FTP, Database queries, Voice, and Video conferencing information, for a period of forty minutes. Our work is to study and compare 
the performance of our UAANET when it uses the reactive routing protocol AODV, and proactive routing protocol OLSR. As shown in the Figure 3.

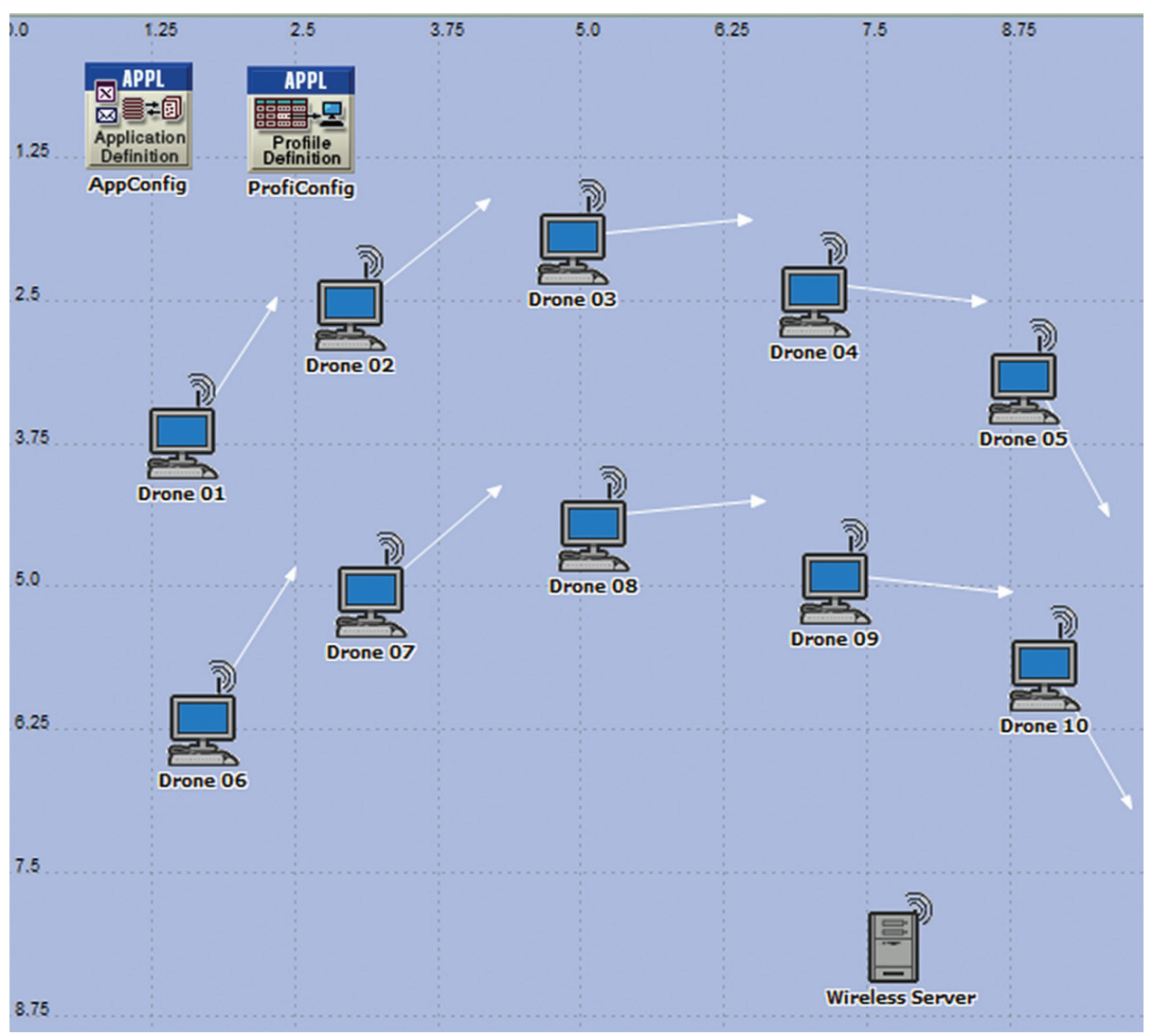

Fig. 3. The UAANET drones network simulation senario

\subsection{Routing of traffic based on the HTTP protocol}

The HTTP traffic sent. Is the average of the number of bytes/sec sent in the UAANET drone network [7], using an application based on the HTTP protocol. The curve that represent the HTTP protocol is in the first position it starts with $1900 \mathrm{bytes} / \mathrm{sec}$ then it make a fast diminution to take $200 \mathrm{bytes} / \mathrm{sec}$ at 01:38 pm, after that it continue the diminution slowly to have 190 bytes/sec at the end of the simulation. For the curve that represent the OLSR protocol it stats with $1200 \mathrm{bytes} / \mathrm{sec}$, then it makes a diminution to have 200 bytes/sec at 01:33:20 pm, then it continue the diminution slowly to have 185 bytes/sec toward the end of the simulation. As shown in the Figure 4. 


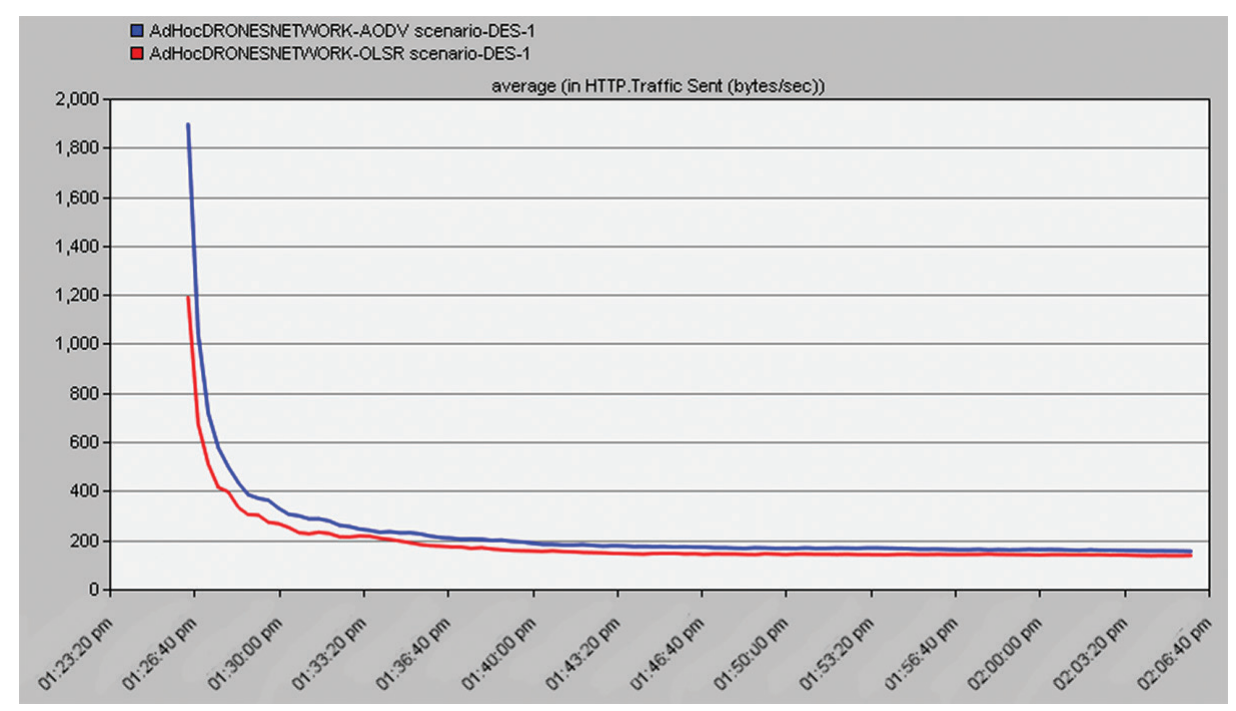

Fig. 4. The average of the HTTP traffic sent (bytes/sec)

\subsection{Routing of traffic for database queries}

Is the amount of information communicated between the drones and the database in bytes/sec. The curve that represents the OLSR protocol is in the first position it takes $16000 \mathrm{bytes} / \mathrm{sec}$ at 01:27 pm, then it decrease and increase to have $10000 \mathrm{bytes} / \mathrm{sec}$ at $01: 31 \mathrm{pm}$, after that it makes a diminution to have $800 \mathrm{bytes} / \mathrm{sec}$ at the end of the simulation. For the curve that represent the AODV protocol, it takes $10000 \mathrm{bytes} / \mathrm{sec}$ at 01:27 pm, then it makes a decrease and an increase to have 8000 bytes $/ \mathrm{sec}$ at 01:31 pm, after that the curve makes a slow diminution to have 700 bytes/sec toward the end of the simulation. As shown in the Figure 5.

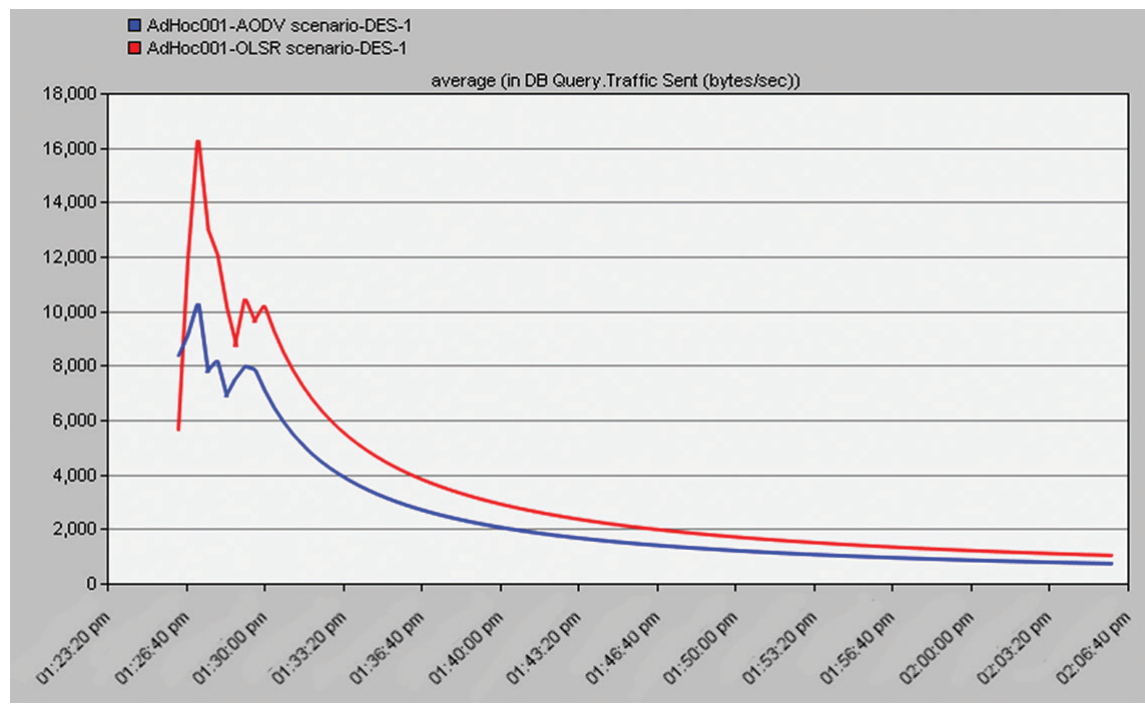

Fig. 5. The data base traffic sent (bytes/sec) 


\subsection{Routing of traffic for a voice application}

The voice packet end to end delay. It the average of the time taken for a voice packet to be transmitted across the network from one drone to the other. The curve that represent the AODV protocol is in the first position it stars with $1.45 \mathrm{sec}$ then it increase in a variable way to have $1.62 \mathrm{sec}$ toward the end of the simulation. For the curve that represent the AODV protocol start at 0.6 second, then it increase 1.58 second at 01:33:20 pm, after that it makes a diminution in a variable way to have $1.4 \mathrm{sec}$ at the end of the simulation. As shown in the Figure 6.

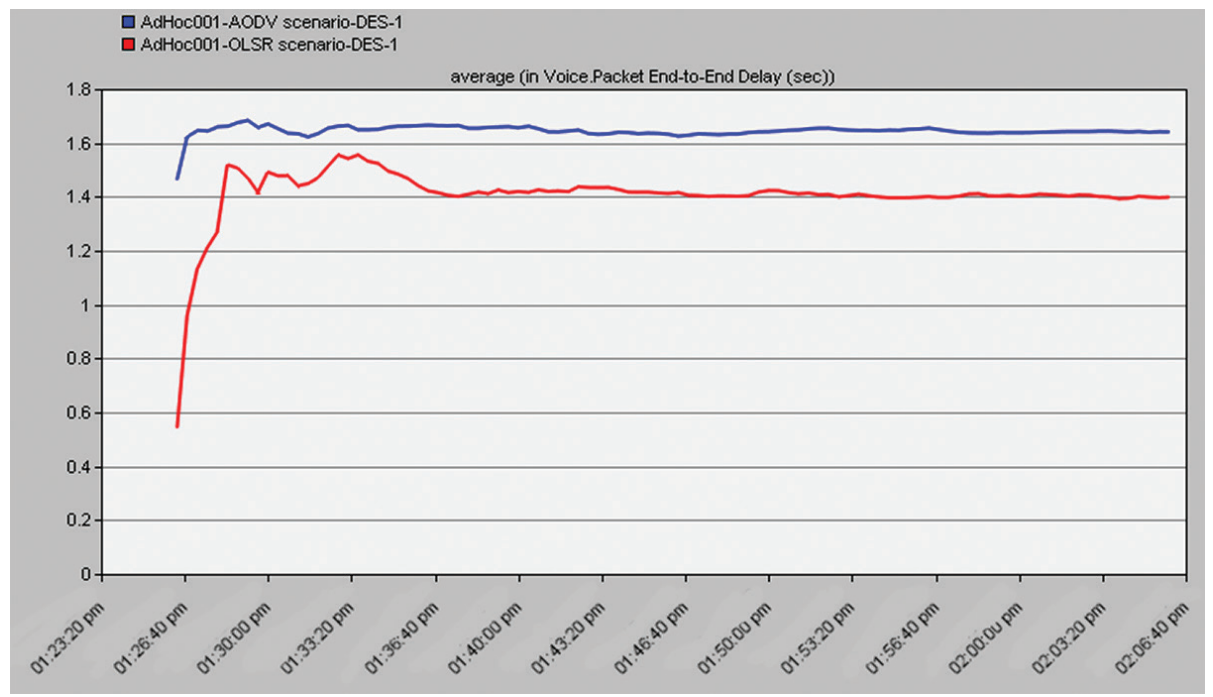

Fig. 6. The average of the voice packet end to end delay (sec)

\subsection{Routing of traffic for a video conferencing application}

Is the amount of video conference information exchanged between the drones during the simulation in bytes/sec. The curve that represent the AODV protocol is in the first position it starts at 1250000 bytes/sec at 01:26:40 pm, then it increase to reach 2400000 bytes/sec at 01:36:40 pm, and stays on this value until the end of the simulation. For the curve that represent the OLSR protocol it start at 850000 bytes/ $\mathrm{sec}$, and make an increase to have $2000000 \mathrm{bytes} / \mathrm{sec}$ at $01: 32 \mathrm{pm}$, then it continue the increase slowly to have 205000 bytes/sec at the end of the simulation. As shown in the Figure 7. 


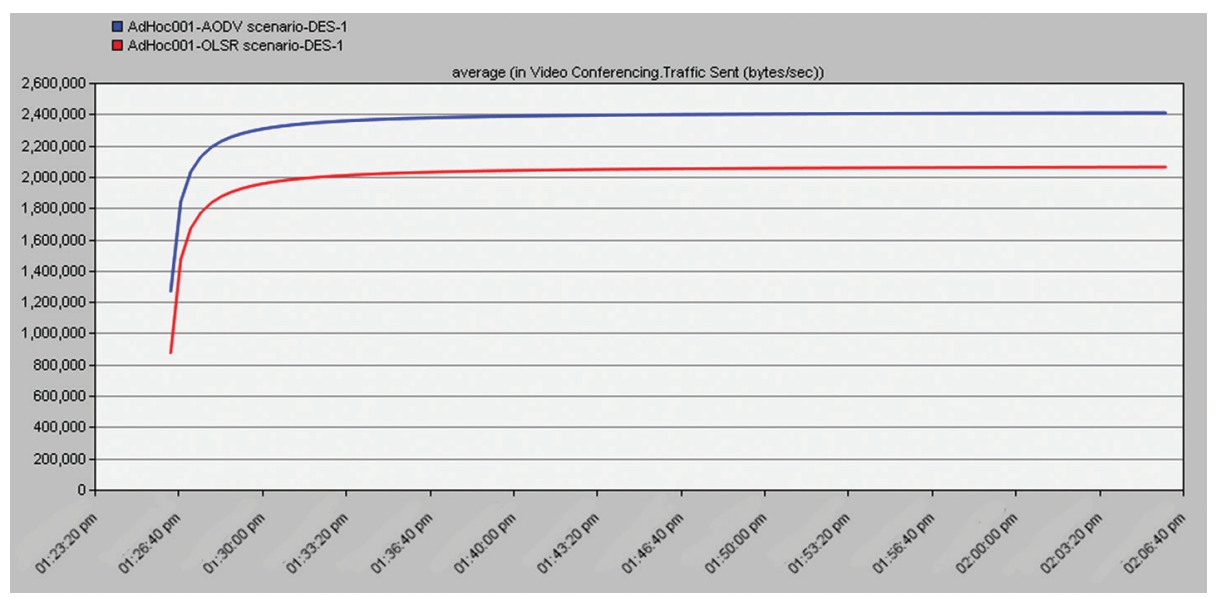

Fig. 7. The average of video conferencing traffic sent (bytes/sec)

\section{Conclusion}

In our work we have talked about the drone fleet that allows autonomous coordination between multiple drones to improve the capacity of the UAS system. The communication between the drones is done through a given communication network UAANET which must guarantee the delivery of the data, while adapting dynamically to the conditions of the implementation of a fleet of drones. To use the UAANET network, it is necessary to set up a dynamic routing protocol that will allow all nodes to communicate. Different types of routing protocols have been proposed in the literature. We have applied in our UAANET drone network, two different type protocols, the AODV protocol and the OLSR protocol, and the performance analysis gives the following results: For routing of a HTTP traffic, the AODV protocol is better than the OLSR protocol. For communication with the database, the OLSR protocol is better than the AODV protocol. For the routing of a voice or a video conference application the AODV protocol is better than the OLSR protocol.

\section{References}

[1] HEUVEL, Milan van den et NYS, Jannes. Communication coordination in network controllability. arXiv preprint arXiv:2105.04164, 2021.

[2] ISSA, Ghassan, HUSSAIN, Shakir M., et ALBAHADILI, Hussain. Unified m-learning model through interactive education satellite: a proposal for an Arab Homeland education satellite. International Journal of Interactive Mobile Technologies (iJIM), 2011, vol. 5, no 2, p. 26-33. https://doi.org/10.3991/ijim.v5i2.1600

[3] HASHIMOTO, Hirofumi, WANG, Weile, DUNGAN, Jennifer L., et al. New generation geostationary satellite observations support seasonality in greenness of the Amazon evergreen forests. Nature Communications, 2021, vol. 12, no 1, p. 1-11. https://doi.org/10.1038/ $\underline{\mathrm{s} 41467-021-20994-\mathrm{y}}$ 
[4] CRISP, Nicholas H., ROBERTS, Peter CE, LIVADIOTTI, Sabrina, et al. In-orbit aerodynamic coefficient measurements using SOAR (Satellite for Orbital Aerodynamics Research). Acta Astronautica, 2021, vol. 180, p. 85-99. https://doi.org/10.1016/j.actaastro.2020.12.024

[5] NGUYEN, H. T., TUAN, H. D., NIYATO, D., et al. Improper Gaussian signaling for D2D communication coexisting MISO cellular networks. IEEE Transactions on Wireless Communications, 2021. https://doi.org/10.1109/TWC.2021.3065961

[6] ZEMRANE H., BADDI Y., et HASBI A. Internet of things Ad hoc drones ecosystem. Procedia Computer Science, 2020, vol. 175, p. 716-722. https://doi.org/10.1016/j.procs. 2020.07.106

[7] RAGAB, Ahmed Refaat. A new classification for Ad-hoc network. iJIM, 2020, vol. 14, no 14, p. 215. https://doi.org/10.3991/ijim.v14i14.14871

[8] ZEMRANE, Hamza, BADDI, Youssef, et HASBI, Abderrahim. Mobile Ad hoc network routing protocols for intelligent transportation systems. International Journal of Smart Security Technologies (IJSST), 2021, vol. 8, no 1, p. 35-48. https://doi.org/10.4018/ IJSST.2021010103

[9] ZEMRANE, Hamza, BADDI, Youssef, et HASBI, Abderrahim. Mobile Ad hoc networks for intelligent transportation system: comparative analysis of the routing protocols. Procedia Computer Science, 2019, vol. 160, p. 758-765. https://doi.org/10.1016/j.procs.2019.11.014

[10] ZEMRANE, Hamza, BADDI, Youssef, et HASBI, Abderrahim. Internet of things Ad Hoc drones ecosystem. Procedia Computer Science, 2020, vol. 175, p. 716-722. https://doi. org/10.1016/j.procs.2020.07.106

[11] HUSSEIN A., EL-RABAIE S., et EL-MASHED M. G. Proactive discovery protocol with security enhancement for D2D communication system. Multimedia Tools and Applications, 2020, pp. 1-20. https://doi.org/10.1007/s11042-020-09799-1

[12] ZEMRANE, Hamza, BADDI, Youssef, et HASBI, Abderrahim. VOIP in MANETs based on the routing protocols OLSR and TORA. In: Advances on Smart and Soft Computing. Springer, Singapore, 2021, p. 443-453. https://doi.org/10.1007/978-981-15-6048-4 39

[13] BABU, C. Naveeth et PRAKASH, V. S. Enhanced medical monitoring wireless sensors networks using proposed greedy multipoint relays protocol algorithm. In: Applications of Artificial Intelligence in Engineering, p. 827.

[14] KHAIRI, Teaba WA, AL-ZUBIDI, Azhar F., et AHMED, Ehsan Qahtan. Modified multipath routing protocol applied on Ns3 Dcell network simulation system. International Journal of Interactive Mobile Technologies, 2021, vol. 15, no 10. https://doi.org/10.3991/ijim. v15i10.22703

[15] ZEMRANE, Hamza, BADDI, Youssef, et HASBI, Abderrahim. IoT EHealth ecosystem based on the Universal Mobile Telecommunications System. 2020. https://doi. org $/ 10.1145 / 3368756.3368981$

\section{Authors}

Hamza Zemrane had a Master's degree in Networks and Computer Systems from the Faculty of Science and Technology of Settat, Hassan First University, and continued his Doctoral thesis at the Mohammadia School of Engineer, in at the Mohammed V University in Rabat, Research Structure: Computer Network, Modeling, and ELearning, he also taught at the Faculty of Science and Technology of Settat, at the level of the Methematic and Computer Science Department (Email: zemranehamza93@gmail.com). 
Pr. Youssef Baddi has a Doctorate in Computer Science from the National School of Computer Science and Systems Analysis, University Mohamed 5 in Rabat, he is also a Professor at the Chouaib Doukali University, at the Higher School of Technology, he is also Director of the Laboratory: STIC (Email: baddi.y@ucd.ac.ma).

Pr. Abderrahim Hasbi is a Professor at the Mohammadia School of Engineer, in at the Mohammed V University in Rabat, permanent researcher at the Research Structure: Computer Network, Modeling, and ELearning, he is also the thesis director (Email: ahasbi@gmail.com).

Article submitted 2020-10-12. Resubmitted 2021-07-12. Final acceptance 2021-07-13. Final version published as submitted by the authors. 